\title{
Addendum to the paper: Nonlinear gradient estimates for elliptic equations in quasiconvex domains
}

\author{
Sun-Sig Byun $^{1}$ - Hun Kwon ${ }^{1}$ • Hyoungsuk So ${ }^{1}$. \\ Lihe Wang ${ }^{2}$
}

Received: 13 April 2015 / Published online: 8 May 2015

(C) Springer-Verlag Berlin Heidelberg 2015

In the above article [1] the funding information was missing. Please find it below.

Acknowledgments S. Byun was supported by Basic Science Research Program through the National Research Foundation of Korea (NRF) funded by the Ministry of Education, Science and Technology (MEST) (2012-047030).

\section{Reference}

1. Byun, S.-S., Kwon, H., So. H., Wang, L.: Nonlinear gradient estimates for elliptic equations in quasiconvex domains. Calc. Var. (2015). doi:10.1007/s00526-015-0830-5

Communicated by L. Caffarelli.

$\bowtie$ Hyoungsuk So

scor1@snu.ac.kr

Sun-Sig Byun

byun@snu.ac.kr

Hun Kwon

kwonhx@snu.ac.kr

Lihe Wang

lwang@math.uiowa.edu

1 Department of Mathematical Science, Seoul National University, Seoul 151-747, Republic of Korea

2 Department of Mathematics, University of Iowa City, Iowa City, IA 52242, USA 